\title{
A Likelihood Ratio Test for Homogeneity in Circular Data
} Juan José Fernández-Durán ${ }^{1 *}$ and María Mercedes Gregorio-Domínguez ${ }^{2}$

${ }^{1}$ Department of Statistics, División Académica de Actuaría, Estadística y Matemáticas, Instituto Tecnológico Autónomo de México ${ }^{2}$ Department of Actuarial Science, División Académica de Actuaría, Estadística y Matemáticas, Instituto Tecnológico Autónomo de México

\begin{abstract}
Testing for the homogeneity of density functions of circular random variables is useful in many settings including the study of wind patterns, paleocurrents trends, the seasonality in human-related events such as homicides and suicides and the seasonality in the appearance of diseases. In this paper, we considered that the density functions are members of the flexible family of circular distributions based on nonnegative trigonometric (Fourier) sums (series) developed by Fernandez-Duran [1]. We constructed a test based on the likelihood ratio and we applied the proposed test to simulated and real datasets.
\end{abstract}

Keywords: Circular data; Nonnegative fourier series

\section{Introduction}

If $\Theta$ is a circular random variable, $\Theta \in(0,2 \pi)$, then the circular density function based on nonnegative trigonometric sums (the NNTS density), developed by Fernandez-Duran [1] and based on Fejer [2], is expressed as

$$
f(\theta ; M, \underline{c})=\left\|\sum_{k=0}^{M} c_{k} e^{i k \theta}\right\|^{2}=\sum_{k=0}^{M} \sum_{l=0}^{M} c_{k} \bar{c}_{l} e^{i(k-l) \theta}
$$

where $i=\sqrt{-1}, C_{k}$ are the complex numbers $C_{k}=C_{r k}+I c_{c k}$ for $k=0, \ldots, M$, and $\bar{c}_{k}=c_{r k}-i c_{c k}$ is the conjugate of $C_{k}$. The following constraint in the $c$ parameter is imposed to $f(\theta ; M, \underline{c})$ to integrate one:

$$
\sum_{k=0}^{M}\left\|c_{k}\right\|^{2}=\frac{1}{2 \pi}
$$

With $c_{c 0}=0$ and $c_{r 0} \geq 0$, i.e., $c_{0}$ is a nonnegative real number. The total number of $c$ free parameters is equal to $2 M$. Also, $M$ is the total number of terms in the sum that defines the NNTS density, which is equal to the maximum number of the modes of the density and is an additional unknown parameter.

Equivalently, the NNTS density can be expressed as

$$
f(\theta ; M, \underline{c})=\frac{1}{2 \pi}+\frac{1}{\pi} \sum_{k=1}^{M}\left(a_{k} \cos (k \theta)+b_{k} \sin (k \theta)\right)
$$

where $a_{k}-i b_{k}=2 \sum_{v=0}^{M-k} c_{v+k} \bar{c}_{v}$ for $k=1,2, \ldots, M$. Note that for the NNTS family, the $k$-th trigonometric moment is equal to $E\left(e^{i k \theta}\right)=a_{k}+i b_{k}$. The case $M=0$ corresponds to a uniform circular density on $(0,2 \pi)$. The NNTS family of circular distributions is very flexible to model datasets that present multimodality and/or skewness.

The accumulated distribution function of an NNTS density is easily calculated as:

$$
\begin{aligned}
& F(\theta ; M, \underline{c})=\int_{0}^{\theta} f(s ; M, \underline{c}) d s=\frac{\theta}{2 \pi}+ \\
& \sum_{k=1}^{M} \frac{a_{k}}{k} \sin (k \theta)+\frac{b_{k}}{k}(1-\cos (k \theta)) .
\end{aligned}
$$

The original support of the NNTS circular distribution is the interval $(0,2 \pi)$, but in applications that are related to the occurrence of events over time, it is more common to use as support the interval $(0,1)$. In that case, we transform the variable $\Theta$ to the variable $T$ by $T=\frac{\Theta}{2 \pi}$ with the density function

$$
f_{T}(t ; M, \underline{c})=1+2 \sum_{k=1}^{M} a_{k} \cos (2 \pi k t)+b_{k} \sin (2 \pi k t)
$$

and the distribution function

$$
F_{T}(t ; M, \underline{c})=t+\frac{1}{\pi} \sum_{k=1}^{M} \frac{a_{k}}{k} \sin (2 \pi k t)+\frac{b_{k}}{k}(1-\cos (2 \pi k t))
$$

For $t \in(0,1)$. In the case that $M=0$ the NNTS distribution is equivalent to a uniform distribution and $f_{T}(t ; M=1)=1$ and $f_{T}(t ; M=0)$ $=t$ for $t \in(0,1)$.

For the case of grouped data $n_{1}, n_{2}, \ldots, n_{R}$ where $n_{i}$ is the total number of the occurrences of the event of interest in the $i$-th interval, $\left(R_{i-l}, R_{i}\right)$ in which $(0,1)$ has been partitioned, $i=0,1, . ., R$, we consider that there are $R$ such intervals. For example, in the case of using a year as the unit of time and months as the intervals, $R_{i-1}$ and $R_{i}$ are the beginning and end, respectively, of the $i$-th month in yearly terms and $R=12$. In the case of grouped data, the likelihood function, $L\left(M, \underline{c} \mid n_{1}, n_{2}, \ldots, n_{R}\right)$, is given by

$$
\begin{aligned}
& L\left(M, \underline{c} \mid n_{1}, n_{2}, \ldots, n_{R}\right)=\prod_{k=1}^{R}\left(F_{T}\left(R_{k}\right)-F_{T}\left(R_{k-1}\right)\right)^{n_{k}} \\
& =\prod_{k=1}^{R}\left(\begin{array}{l}
\left.R_{k}-R_{k-1}+\frac{1}{\pi} \sum_{k=1}^{M} \frac{a_{k}}{k}\left(\sin \left(2 \pi k R_{k}\right)-\sin \left(2 \pi k R_{k-1}\right)\right)+\right)^{n_{k}} \\
\frac{b_{k}}{k}\left(\cos \left(2 \pi k R_{k-1}\right)-\cos \left(2 \pi k R_{k}\right)\right)
\end{array}\right)
\end{aligned}
$$

Additional properties of the NNTS circular models are presented by Fernandez-Duran $[1,3]$. The maximum likelihood estimates of the parameters of the NNTS model are obtained using an efficient Newtonlike algorithm that was developed by Fernandez-Duran [4] and implemented in the statistical software R [5] in the library CircNNTSR $[6,7]$.

Previous work on homogeneity tests for circular data includes that of Mardia and Spurr [8] for $l$-modal and axial von Mises distributions with modes separated by a constant arc. In addition, Mardia [9] developed tests against shift-type alternatives, based on uniform scores as an extension of Wheeler and Watson [10]. In particular, Rao's test of homogeneity $[11,12]$ tests for the equality of the mean directions and dispersions of $N$ circular populations by considering the means of the

*Corresponding author: Fernandez-Duran JJ, Department of Statistics, División Académica de Actuaría, Estadística y Matemáticas, Instituto Tecnológico Autónomo de México, Rio Hondo No. 1, Col. Progreso Tizapan, C.P.01080, México D.F., México, Tel: (5255) 562838 39; Fax: (5255) 562840 86; E-mail: jfdez@itam.mx

Received October 14, 2010; Accepted November 22, 2010; Published November 24, 2010

Citation: Fernandez-Duran JJ, Gregorio-Dominguez MM (2010) A Likelihood Ratio Test for Homogeneity in Circular Data. J Biomet Biostat 1:107. doi:10.4172/21556180.1000107

Copyright: (c) 2010 Fernandez-Duran JJ, et al. This is an open-access article distributed under the terms of the Creative Commons Attribution License, which permits unrestricted use, distribution, and reproduction in any medium, provided the original author and source are credited. 
Citation: Fernandez-Duran JJ, Gregorio-Dominguez MM (2010) A Likelihood Ratio Test for Homogeneity in Circular Data. J Biomet Biostat 1:107. doi:10.4172/2155-6180.1000107

Page 2 of 9

cosine and sine values, $\bar{C}_{j}=n_{j}^{-1} \sum_{k=1}^{n_{j}} \cos \left(\theta_{k j}\right)$ and $\bar{S}_{j}=n_{j}^{-1} \sum_{k=1}^{n_{j}} \sin \left(\theta_{k j}\right)$ for $j=1, \ldots, N$, by comparing the values of $T_{j}=\frac{\bar{S}_{j}}{\bar{C}_{j}}$ for testing the equality of the mean directions, and $U_{j}=\bar{C}_{j}^{2}+\bar{S}_{j}^{2}$ for testing the equality of the dispersions [13]. Watson's two-sample test of homogeneity uses the squared differences between the sample distribution functions as test statistic [14]. Tests for the equality of the mean directions and dispersion parameters of von Mises populations that were developed by Mardia [9], Watson and Williams [15], Stephens [16], Watson [17], and Fisher [18] are similar to the homogeneity tests. Similarly, Harrison et al. $[19,20]$, Stephens $[21,22]$ have developed two-way and multi-way ANOVAs for circular data. Recently, Grimshaw et al. [23], Chen et al. [24], and Fu et al. [25] developed homogeneity tests for mixtures of von Mises distributions using likelihood ratio tests. In contrast to previous work that used von Mises or mixtures of von Mises densities as the population densities, the main objective of the present paper was to develop a likelihood ratio test for the homogeneity of circular populations by considering their density functions as members of the flexible NNTS family for ungrouped and grouped observations.

This paper is divided into five sections, including this introduction. The second section outlines the development of the likelihood ratio test for homogeneity. In the third section, the proposed test is applied to simulated and real datasets. The fourth section includes a simulation exercise to study the type I error and power of the proposed test. Lastly, in the fifth section, the conclusions of the present work are presented.

\section{Likelihood ratio homogeneity test}

Ungrouped observation: Let $\underline{\theta}_{k}=\left(\theta_{k 1}, \theta_{k 2}, \ldots, \theta_{k n_{k}}\right)$ for $k=1, \ldots, N$ be independent random samples from $N$ different continuous circular populations, and let $F_{k}(\theta)$ and $f_{k}(\theta)$ be their respective distribution (density) functions. A test of homogeneity has a null hypothesis given as $H_{0}: F_{1}=F_{2}=\ldots=F_{N}\left(H_{0}: f_{1}=f_{2}=\ldots=f_{N}\right)$ and an alternative hypothesis given as $H_{a}: F_{1}, \ldots, F_{N}$ are not all the same $\left(H_{a}: f_{1}, \ldots, f_{N}\right.$ are not all the same). By considering that for $k=1, \ldots, N, F_{k}\left(f_{k}\right)$ is a member of the NNTS family of the circular distributions with parameters $M_{k}$ and $c_{k}$, a test for homogeneity can be constructed by considering the likelihood ratio, This ratio is defined as

$$
\Lambda=\frac{\max \left\{L_{0}\left(M_{0}, \underline{c}_{0} \mid \underline{\theta}_{1}, \ldots, \underline{\theta}_{N}\right)\right\}}{\max \left\{L\left(M_{0}, \underline{c} \mid \underline{\theta}_{1}, \ldots, \underline{\theta}_{N}\right)\right\}}=\frac{L\left(M_{0}, \underline{c}_{0} \mid \underline{\theta}_{1}, \ldots, \underline{\theta}_{N}\right)}{L\left(M_{0}, \underline{c}_{1} \mid \underline{\theta}_{1}, \ldots, \underline{\theta}_{N}\right)}
$$

where $L\left(M_{0}, \underline{c}_{0} \mid \underline{\theta}_{1}, \ldots, \underline{\theta}_{N}\right)$ is the maximum of the likelihood function over the restricted parameter space specified by $H_{0}$, and $\underline{c}_{0}$ is the maximum likelihood estimate under the null hypothesis. Note that we are considering that $M_{k}=M_{0}$ for $k=1, \ldots, N$ and that the value of $M_{0}$ is specified before applying the test. The researcher can select a value for $M_{0}$ by considering the maximum number of modes in all of the considered populations (taking into account the sample sizes in each population) because the NNTS models are nested with respect to the increasing values of the parameter $M$. Now, $L\left(M_{0}, \underline{c} \mid \underline{\theta}_{1}, \ldots, \underline{\theta}_{N}\right)$ is the maximum of the likelihood function over the complete parameter space, and $\underline{c}=\left(c_{1}, \ldots, \underline{c}_{N}\right)$ are the unrestricted maximum likelihood estimates that corresponds to the unrestricted maximum likelihood estimate for the parameter $\underline{c}$ in each of the $N$ considered circular populations. The likelihood function for the ungrouped data under the null hypothesis is calculated as

$$
L_{0}\left(M_{0}, \underline{c}_{0} \mid \underline{\theta}_{1}, \ldots, \underline{\theta}_{N}\right)=\prod_{k=1}^{N} \prod_{i=1}^{n_{k}} f\left(\theta_{i k} \mid M_{0}, \underline{c}_{0}\right)
$$

where $f\left(\theta_{i k} \mid M_{0}, \underline{c}_{0}\right)$ is the common NNTS circular density function

\begin{tabular}{|c|c|c|c|c|c|c|}
\hline$M_{0}$ & Population 1 & Population 2 & Populations 1 and 2 & $-2 \ln (\Lambda)$ & d.f. & \\
\hline 0 & -91.8939 & -91.8939 & -183.7877 & & \\
\hline 1 & -72.8523 & -71.1008 & -144.1506 & 0.3949 & \\
\hline 2 & -68.7839 & -66.1711 & -135.5405 & 1.1710 & 2 \\
\hline 3 & -68.2188 & -65.0110 & -134.3438 & 2.2281 & 0.8208 \\
\hline 4 & -65.6811 & -64.7347 & -133.2813 & 5.7310 & 6 \\
\hline 5 & -64.6047 & -64.6029 & -132.6212 & 6.8271 & 0.8829 \\
\hline 6 & -63.9279 & -64.4850 & -131.9476 & 7.0695 & 10 \\
\hline
\end{tabular}

Table 1: Results of the NNTS likelihood ratio test for homogeneity when applied to two samples of 50 data points from a von Mises population with $\mu=3.1962$ and $\kappa=$

\begin{tabular}{|c|c|c|c|c|c|c|c|c|c|c|c|}
\hline$M_{0}$ & Pop. 1 & Pop. 2 & Pop. 3 & Pop. 4 & Pop. 5 & Pop. 6 & Pop. 7 & Pops. 1 to 7 & $-2 \ln (\Lambda)$ & d.f. & $\chi^{2} p$-value \\
\hline 0 & -91.8939 & -91.8939 & -91.8939 & -91.8939 & -91.8939 & -91.8939 & -91.8939 & -643.2570 & & & \\
\hline 1 & -90.7435 & -90.8332 & -91.8541 & -91.8634 & -91.2191 & -91.6372 & -91.8033 & -642.4010 & 4.8942 & 12 & 0.9614 \\
\hline 2 & -89.6939 & -89.4777 & -91.3742 & -89.0410 & -91.2099 & -91.2177 & -88.7269 & -641.9138 & 22.3450 & 24 & 0.5587 \\
\hline 3 & -88.4427 & -89.3987 & -89.9833 & -88.9220 & -90.0088 & -88.3413 & -86.7960 & -640.5025 & 37.2196 & 36 & 0.4127 \\
\hline 4 & -86.8474 & -88.8507 & -89.9685 & -86.4008 & -89.3175 & -87.7075 & -86.7055 & -639.4287 & 47.2615 & 48 & 0.5030 \\
\hline 5 & -84.6353 & -88.3876 & -86.1472 & -85.5370 & -87.7413 & -87.0995 & -86.5498 & -637.6810 & 63.1670 & 60 & 0.3651 \\
\hline 6 & -84.1664 & -83.5998 & -85.8473 & -85.5001 & -87.7211 & -85.1840 & -83.3282 & -636.9619 & 83.2302 & 72 & 0.1720 \\
\hline
\end{tabular}
1.5510 .

\begin{tabular}{|c|c|c|c|c|c|c|}
\hline$M_{0}$ & $1951-1970$ & $1971-2008$ & $1951-2008$ & $-2 \ln (\wedge)$ & d.f. & $x^{2} p$-value \\
\hline 0 & -360.2239 & -766.3947 & -1126.6190 & & & \\
\hline 1 & -250.9487 & -530.7715 & -781.7269 & 0.0134 & 2 & 0.9933 \\
\hline 2 & -213.7020 & -456.5898 & -671.3008 & 2.0180 & 4 & 0.7324 \\
\hline 3 & -203.8207 & -431.7584 & -636.8067 & 2.4552 & 6 & 0.8734 \\
\hline 4 & -196.9491 & -424.7851 & -626.3378 & 9.2072 & 8 & 0.3251 \\
\hline 5 & -191.2163 & -419.8673 & -615.7329 & 9.2986 & 10 & 0.5040 \\
\hline 6 & -191.1102 & -415.6410 & -612.2737 & 11.0450 & 12 & 0.5251 \\
\hline 7 & -190.5867 & -415.3392 & -611.7719 & 11.6920 & 14 & 0.6310 \\
\hline 8 & -189.7132 & -414.9694 & -611.6512 & 13.9372 & 16 & 0.6034 \\
\hline 9 & -189.3480 & -413.3788 & -610.6316 & 15.8096 & 18 & 0.6058 \\
\hline 10 & -188.1896 & -413.3687 & -610.3693 & 17.6220 & 20 & 0.6123 \\
\hline
\end{tabular}

Table 2: Results of the NNTS likelihood ratio test for homogeneity when applied to seven samples of 50 data points from a circular uniform distribution.

\begin{tabular}{|c|c|c|c|c|c|c|c|c|c|c|c|c|c|}
\hline Season & & & & & & & & & & & & & \\
\hline Winter & 50 & 120 & 190 & 210 & 220 & 250 & 260 & 290 & 290 & 320 & 320 & 340 & \\
\hline Spring & 0 & 20 & 40 & 60 & 160 & 170 & 200 & 220 & 270 & 290 & 340 & 350 & \\
\hline Summer & 10 & 10 & 20 & 20 & 30 & 30 & 40 & 150 & 150 & 150 & 170 & 190 & 290 \\
\hline Autumn & 30 & 70 & 110 & 170 & 180 & 190 & 240 & 250 & 260 & 260 & 290 & 350 & \\
\hline
\end{tabular}

Table 3: Results of the NNTS homogeneity test for the hurricane data.

Table 4: Wind directions in degrees at Gorleston, England [26]. 
for all of the observations in all of the considered populations. The likelihood under the complete parameter space corresponds to

$$
\begin{aligned}
& L\left(M_{0}, \underline{c} \mid \underline{\theta}_{1}, \ldots, \underline{\theta}_{N}\right)=\prod_{k=1}^{N} L_{k}\left(M_{0}, \underline{c}_{k} \mid \underline{\theta}_{k}\right)= \\
& \prod_{k=1}^{N} \prod_{i=1}^{n_{k}} f\left(\theta_{i k} \mid M_{0}, \underline{c}_{k}\right)
\end{aligned}
$$

where $f\left(\theta_{i k} \mid M_{0}, \underline{c}_{k}\right)$ corresponds to the NNTS circular density of the $k$-th population, with parameters $M_{0}$ and $\underline{c}_{k}$. Maximizing $L\left(M_{0}, \underline{c} \mid \underline{\theta}_{1}, \ldots, \underline{\theta}_{N}\right)$ is equivalent to maximizing each of the $L_{k}\left(\underline{\theta}_{k} \mid M_{0}, \underline{c}_{k}\right)$ likelihood functions for each population, with respect to the corresponding $\underline{\mathcal{c}}_{k}$ parameters for $k=1, \ldots, N$.

By the likelihood asymptotic theory, $-2 \ln (\Lambda)$ converges to a chi-squared distributed random variable with a number of degrees of freedom that is equal to the difference in the number of the free parameters in the unrestricted and restricted parameter spaces.

Grouped observations: In the case that the observed data consists of grouped observations, i.e., only the total number of occurrences of the circular random variables in the different intervals is observed, then, the likelihood function for the null hypothesis of homogeneity is calculated as

$$
L\left(M_{0}, \underline{c}_{0} \mid \underline{n}_{1}, \ldots, \underline{n}_{N}\right)=\prod_{k=1}^{N} \prod_{i=1}^{I_{k}}\left(\begin{array}{l}
F\left(R_{k i} \mid M_{0}, \underline{c}_{0}\right)- \\
F\left(R_{k, i-1} \mid M_{0}, \underline{c}_{0}\right)
\end{array}\right)^{n_{k i}}
$$

where $n_{k i}$ is the total number of observations of population $k$ in the $i$-th interval in population $k,\left(R_{k, i-1}, R_{k i}\right)$. The total number of grouping intervals for population $k$ is denoted by $I_{k}$. Note that the grouping intervals may be different for each population. The likelihood function for the whole parameter space is given by

$$
\begin{aligned}
& L\left(M_{0}, \underline{c} \mid \underline{n_{1}}, \ldots, \underline{n}_{N}\right)=\prod_{k=1}^{N} L_{k}\left(M_{0}, \underline{c}_{k} \mid \underline{n}_{k}\right) \\
& =\prod_{k=1}^{N} \prod_{i=1}^{I_{k}}\left(F\left(R_{k i} \mid M_{0}, \underline{c}_{k}\right)-F\left(R_{k, i-1} \mid M_{0}, \underline{c}_{k}\right)\right)^{n_{i k}}
\end{aligned}
$$

In practice, the grouping intervals are commonly the same for all of the observed populations.

\section{Examples}

\section{Ungrouped simulated data}

Simulated von mises data: A random sample of 100 observations from a von Mises density, with a location parameter $\mu=3.1962$ and a dispersion parameter $=1.5510$, was generated. We divided the dataset in two. The first dataset included the first 50 observations, and the second included the last 50 observations. The results of applying the NNTS homogeneity likelihood ratio test to these datasets are included in Table 1. The NNTS models of order $M_{0}=0,1, \ldots, 6$ were fitted to each dataset of 50 observations (Population 1 and Population 2) and to the complete dataset of 100 observations (Populations 1 and 2). The columns in Table 1 contain the values of the maximized log-likelihood, the observed value of the likelihood ratio statistic $(-2 \ln (\Lambda))$, the number of the degrees of freedom (d.f.), and the corresponding p-values when comparing the observed test statistic with the distribution of a chi-squared random variable. For $M_{0}=1,2, \ldots, 6$, the observed values of the NNTS likelihood ratio statistic resulted, as expected, in the decision of not rejecting the null hypothesis of homogeneity.

Simulated circular uniform data: We generated seven random samples of 50 data points from a circular uniform distribution. Table 2 contains the results of the NNTS likelihood ratio test. For all of the considered values of $M_{0}$, the null hypothesis of homogeneity was not rejected.

\section{Ungrouped real data}

Hurricane occurrence data: Recently, there has been debate about whether an increase exists in the number of tropical storms and hurricanes that occur yearly in the North Atlantic Ocean. Instead of analyzing the possibility of an increase in the number of tropical storms and hurricanes, we analyzed whether there is evidence of a change in the start date of the tropical storms and hurricanes. The starting dates of the tropical storms and hurricanes were obtained from http://weather. unisys.com/archive/index.html. This website includes tropical storms and hurricanes that occurred between 1851 and 2008 and is based on the HURDAT database of the National Oceanic and Atmospheric Administration (NOAA). The dates were converted to numbers between 0 and 1 to represent the fraction of the year that elapsed at the starting date of the tropical storm or hurricane. These values were then multiplied by $2 \pi$ to convert them to circular data. Then, we compared the starting dates of the storms that occurred between 1951 and 1970 to those that occurred between 1971 and 2008. Table 3 contains the results of the NNTS homogeneity test. For all of the considered values of $M_{0^{2}}$ we did not reject the null hypothesis of homogeneity.

Wind direction data: Mardia and Jupp [26] analyzed a dataset of the wind directions in degrees at Gorleston, England, between 11 a.m. and 12 p.m. on Sundays in 1968, as classified by seasons. The data is shown in Table 4.

The authors applied a homogeneity test to the data based on the ranks of the angles and the uniformity test of Rayleigh, known as the

\begin{tabular}{|c|c|c|c|c|c|c|c|c|}
\hline$M_{0}$ & Winter & Spring & Summer & Autumn & Combined & $-2 \ln (\wedge)$ & d.f. & $x^{2} p$-value \\
\hline 0 & -22.0545 & -22.0545 & -23.8924 & -22.0545 & -90.0560 & & & \\
\hline 1 & -19.9543 & -21.6284 & -22.1090 & -21.3295 & -89.1455 & 8.2486 & 6 & 0.2205 \\
\hline 2 & -19.5298 & -19.5591 & -18.7057 & -20.6901 & -86.5384 & 16.1075 & 12 & 0.1864 \\
\hline 3 & -18.9205 & -19.3480 & -15.0076 & -20.5220 & -83.9955 & 20.3948 & 18 & 0.3110 \\
\hline 4 & -17.3523 & -18.3262 & -14.5950 & -18.0935 & -83.5365 & 30.3392 & 24 & 0.1738 \\
\hline 5 & -16.9660 & -17.7181 & -11.9490 & -17.0136 & -83.0619 & 38.8305 & 30 & 0.1296 \\
\hline 6 & -16.9440 & -16.5327 & -11.7001 & -16.5227 & -82.8683 & 42.3377 & 36 & 0.2163 \\
\hline
\end{tabular}
uniform scores test [27], to this dataset. They concluded that the null hypothesis was rejected at the $5 \%$ level ( $\mathrm{p}$-value=0.046). We applied the

\begin{tabular}{|c|c|c|c|c|c|c|}
\hline$M_{0}$ & set 1 & set2 & combined set (1 and 2) & $-2 \ln (\wedge)$ & d.f. & $\mathrm{X}^{2} \mathrm{p}$-value \\
\hline 0 & -45.9469 & -191.1392 & -237.0861 & & & \\
\hline 1 & -41.4898 & -176.0237 & -218.5734 & 2.1199 & 2 & 0.3465 \\
\hline 2 & -38.9377 & -169.1353 & -215.9945 & 15.8430 & 4 & 0.0032 \\
\hline 3 & -38.3024 & -168.9135 & -215.8106 & 17.1893 & 6 & 0.0086 \\
\hline 4 & -37.0521 & -168.4393 & -215.4071 & 19.8314 & 8 & 0.0110 \\
\hline 5 & -36.9842 & -168.2582 & -215.3776 & 20.2705 & 10 & 0.0268 \\
\hline 6 & -36.3596 & -168.2576 & -215.3653 & 21.4962 & 12 & 0.0436 \\
\hline
\end{tabular}
NNTS homogeneity test to this dataset, and the results are shown in Table 5.

Table 5: NNTS homogeneity tests: Results for the wind directions dataset [26].

Table 6: NNTS homogeneity tests results for the cross-bed measurements from the Himalayan molasse dataset [29]. 
Citation: Fernandez-Duran JJ, Gregorio-Dominguez MM (2010) A Likelihood Ratio Test for Homogeneity in Circular Data. J Biomet Biostat 1:107. doi:10.4172/2155-6180.1000107

Page 4 of 9

\begin{tabular}{|c|c|c|c|c|c|c|c|c|c|c|c|c|c|}
\hline & Year & Jan & Feb & Mar & Apr & May & Jun & Jul & Aug & Sep & Oct & Nov & Dec \\
\hline Suicides & 2005 & 318 & 335 & 370 & 360 & 428 & 367 & 366 & 386 & 390 & 342 & 331 & 320 \\
\hline & $\%$ & 7.37 & 7.77 & 8.58 & 8.35 & 9.92 & 8.51 & 8.49 & 8.95 & 9.04 & 7.93 & 7.67 & 7.42 \\
\hline & 2007 & 284 & 303 & 416 & 391 & 413 & 392 & 383 & 383 & 350 & 367 & 354 & 355 \\
\hline & $\%$ & 6.47 & 6.90 & 9.47 & 8.90 & 9.41 & 8.93 & 8.72 & 8.72 & 7.97 & 8.36 & 8.06 & 8.08 \\
\hline Homicides & 2005 & 776 & 776 & 819 & 859 & 822 & 889 & 817 & 792 & 837 & 814 & 825 & 881 \\
\hline & $\%$ & 7.83 & 7.83 & 8.27 & 8.67 & 8.30 & 8.97 & 8.25 & 7.99 & 8.45 & 8.22 & 8.33 & 8.89 \\
\hline & 2007 & 595 & 508 & 781 & 775 & 935 & 757 & 740 & 764 & 713 & 768 & 708 & 797 \\
\hline & $\%$ & 6.73 & 5.75 & 8.83 & 8.77 & 10.58 & 8.56 & 8.37 & 8.64 & 8.06 & 8.69 & 8.01 & 9.01 \\
\hline
\end{tabular}

Table 7: Suicides and homicides in Mexico for the years of 2005 and 2007.

\begin{tabular}{|c|c|c|c|c|c|c|}
\hline$M_{0}$ & Suicides 2005 & Homicides 2005 & Suicides and Homicides 2005 & $-2 \ln (\wedge)$ & d.f. & $x^{2} p$-value \\
\hline 0 & -10717.05 & -24620.57 & -35337.62 & & & \\
\hline 1 & -10707.97 & -24620.20 & -35333.37 & 10.40 & 2 & 0.0055 \\
\hline 2 & -10706.07 & -24618.29 & -35331.64 & 14.56 & 4 & 0.0057 \\
\hline 3 & -10705.13 & -24618.17 & -35331.44 & 16.28 & 6 & 0.0123 \\
\hline 4 & -10702.36 & -24614.96 & -35327.69 & 20.74 & 8 & 0.0079 \\
\hline 5 & -10701.89 & -24611.23 & -35324.31 & 22.38 & 10 & 0.0133 \\
\hline 6 & -10701.56 & -24609.14 & -35323.52 & 25.64 & 12 & 0.0121 \\
\hline
\end{tabular}

Table 8: NNTS homogeneity test results: Suicides and homicides in Mexico in 2005

\begin{tabular}{|c|c|c|c|c|c|c|}
\hline$M_{0}$ & Suicides 2007 & Homicides 2007 & Suicides and Homicides 2007 & $-2 \ln (\wedge)$ & d.f. & $x^{2} p$-value \\
\hline 0 & -10907.41 & -21949.65 & -32857.06 & & & \\
\hline 1 & -10896.88 & -21931.98 & -32829.40 & 1.08 & 2 & 0.5827 \\
\hline 2 & -10891.65 & -21903.18 & -32798.34 & 7.02 & 4 & 0.1348 \\
\hline 3 & -10888.55 & -21895.23 & -32790.39 & 13.22 & 6 & 0.0397 \\
\hline 4 & -10886.79 & -21892.36 & -32785.85 & 13.40 & 8 & 0.0988 \\
\hline 5 & -10886.16 & -21883.11 & -32777.17 & 15.80 & 10 & 0.1055 \\
\hline 6 & -10886.10 & -21882.93 & -32777.14 & 16.22 & 12 & 0.1814 \\
\hline
\end{tabular}

Table 9: NNTS homogeneity test results: Suicides and homicides in Mexico in 2007.

In contrast to the uniform score test results of Mardia and Jupp [26], we did not reject the null hypothesis of homogeneity for the considered values of $M_{0}$. This contrast may be due to the small sample sizes. Mardia and Jupp [26] also analyzed this dataset using the von Mises model and concluded that it was not possible to reject the null hypotheses of equal mean directions and equal concentration parameters. Fisher [28] also analyzed this dataset. By applying a uniform score test for the Winter, Spring, and Autumn data, Fisher concluded that there was little evidence that the wind distributions in these three seasons differed.

Himalayan molasse data: Fisher [28] analyzed two samples of cross-bed measurements that were collected from the Himalayan molasse in Pakistan [29]. The first sample consisted of 35 measurements of the Rakhi Nala ripple cross-beds, and the second sample consisted of 104 measurements of the Chaudhwan Zam large bedforms. Fisher suggested that the samples were drawn from von Mises distributions with different shapes but not necessarily with different mean directions and applied a bootstrap method test for different means. Fisher did not find evidence that the mean directions differed. The results of the NNTS homogeneity test are shown in Table 6. For the selected $M_{0}$ values equal to 2 and 3 , the null hypothesis of homogeneity was rejected at the $1 \%$ significance level. For $M_{0}=4,5$ and 6 , the null hypothesis was rejected at the $5 \%$ significance level, and for $M_{0}=1$, the null hypothesis was not rejected because the p-value was equal to 0.3465 . This example illustrates the necessity to select a sensible value for $M_{0}$ before applying the test.

\section{Grouped real data}

Suicides and homicides data: The Mexican Statistical Agency (INEGI) reports the monthly number of suicides and homicides in Mexico every year. To compare the monthly patterns of the number of suicides and homicides, we applied the NNTS homogeneity test for the monthly numbers of suicides and homicides for the years of 2005 and 2007. Table 7 shows the monthly grouped raw data.

Table 8 shows the results of applying the NNTS homogeneity test to the number of suicides and homicides in Mexico in 2005. The null hypothesis of homogeneity was rejected at the $5 \%$ significance level when using $M_{0}=1,2,3,4,5$ and 6 . Note that the case $M_{0}=6$ corresponds to the saturated model.

For the data from the year 2007, Table 9 shows the results of the NNTS homogeneity test where, for $M_{0}=1,2,4$, and 6 the test did not reject the null hypothesis of homogeneity. Only for $M_{0}=3$ was the null hypothesis of homogeneity rejected at the $5 \%$ significance level. The change in the seasonal patterns of suicides and homicides from 2005 to 2007 may be the result of the mis-reporting, over-reporting, or underreporting of these crimes.

\section{Power and type I error of the NNTS test of homogeneity}

To study the power of the NNTS test of homogeneity and its type I error rate, we simulated data from three different models: a uniform

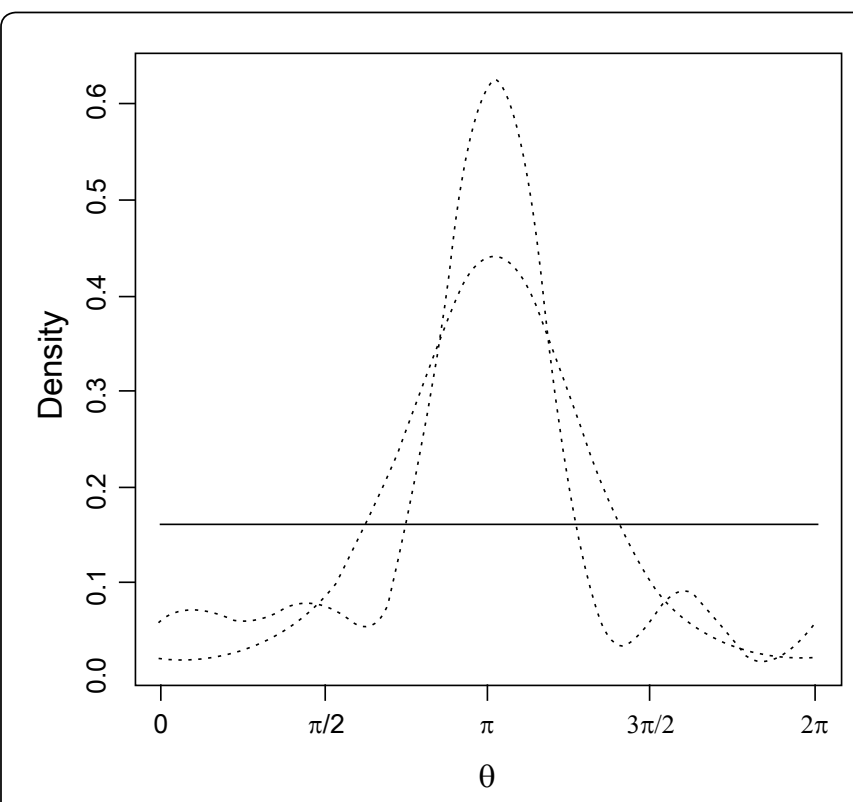

Figure 1: Considered models in the simulation study (Uniform (solid), von Mises (dot dash) and NNTS with $M=4$ (dash)). 
Citation: Fernandez-Duran JJ, Gregorio-Dominguez MM (2010) A Likelihood Ratio Test for Homogeneity in Circular Data. J Biomet Biostat 1:107. doi:10.4172/2155-6180.1000107

Page 5 of 9

\begin{tabular}{|c|c|c|c|c|c|c|c|c|c|c|c|c|c|c|c|}
\hline & & & & & & & & & NNT & est & & & & & \\
\hline Model 1 & $n_{1}$ & Model 2 & $n_{2}$ & $\alpha$ & $M_{0}=1$ & 2 & 3 & 4 & 5 & 6 & 7 & 8 & 9 & 10 & Watson \\
\hline Uniform & 20 & von Mises & 20 & 0.01 & 100 & 100 & 100 & & & & & & & & 100 \\
\hline & & & & 0.05 & 100 & 100 & 100 & & & & & & & & 100 \\
\hline & & & & 0.1 & 100 & 100 & 100 & & & & & & & & 100 \\
\hline & & & 50 & 0.01 & 100 & 100 & 100 & & & & & & & & 100 \\
\hline & & & & 0.05 & 100 & 100 & 100 & & & & & & & & 100 \\
\hline & & & & 0.1 & 100 & 100 & 100 & & & & & & & & 100 \\
\hline & & & 100 & 0.01 & 100 & 100 & 100 & & & & & & & & 100 \\
\hline & & & & 0.05 & 100 & 100 & 100 & & & & & & & & 100 \\
\hline & & & & 0.1 & 100 & 100 & 100 & & & & & & & & 100 \\
\hline & & NNTS & 20 & 0.01 & 100 & 100 & 100 & & & & & & & & 100 \\
\hline & & & & 0.05 & 100 & 100 & 100 & & & & & & & & 100 \\
\hline & & & & 0.1 & 100 & 100 & 100 & & & & & & & & 100 \\
\hline & & & 50 & 0.01 & 100 & 100 & 100 & & & & & & & & 100 \\
\hline & & & & 0.05 & 100 & 100 & 100 & & & & & & & & 100 \\
\hline & & & & 0.1 & 100 & 100 & 100 & & & & & & & & 100 \\
\hline & & & 100 & 0.01 & 100 & 100 & 100 & & & & & & & & 100 \\
\hline & & & & 0.05 & 100 & 100 & 100 & & & & & & & & 100 \\
\hline & & & & 0.1 & 100 & 100 & 100 & & & & & & & & 100 \\
\hline Uniform & 50 & von Mises & 20 & 0.01 & 100 & 100 & 100 & & & & & & & & 100 \\
\hline & & & & 0.05 & 100 & 100 & 100 & & & & & & & & 100 \\
\hline & & & & 0.1 & 100 & 100 & 100 & & & & & & & & 100 \\
\hline & & & 50 & 0.01 & 100 & 100 & 100 & 100 & 100 & 100 & & & & & 100 \\
\hline & & & & 0.05 & 100 & 100 & 100 & 100 & 100 & 100 & & & & & 100 \\
\hline & & & & 0.1 & 100 & 100 & 100 & 100 & 100 & 100 & & & & & 100 \\
\hline & & & 100 & 0.01 & 100 & 100 & 100 & 100 & 100 & 100 & & & & & 100 \\
\hline & & & & 0.05 & 100 & 100 & 100 & 100 & 100 & 100 & & & & & 100 \\
\hline & & & & 0.1 & 100 & 100 & 100 & 100 & 100 & 100 & & & & & 100 \\
\hline & & NNTS & 20 & 0.01 & 100 & 100 & 100 & & & & & & & & 100 \\
\hline & & & & 0.05 & 100 & 100 & 100 & & & & & & & & 100 \\
\hline & & & & 0.1 & 100 & 100 & 100 & & & & & & & & 100 \\
\hline & & & 50 & 0.01 & 100 & 100 & 100 & 100 & 100 & 100 & & & & & 100 \\
\hline & & & & 0.05 & 100 & 100 & 100 & 100 & 100 & 100 & & & & & 100 \\
\hline & & & & 0.1 & 100 & 100 & 100 & 100 & 100 & 100 & & & & & 100 \\
\hline & & & 100 & 0.01 & 100 & 100 & 100 & 100 & 100 & 100 & & & & & 100 \\
\hline & & & & 0.05 & 100 & 100 & 100 & 100 & 100 & 100 & & & & & 100 \\
\hline & & & & 0.1 & 100 & 100 & 100 & 100 & 100 & 100 & & & & & 100 \\
\hline Uniform & 100 & von Mises & 20 & 0.01 & 100 & 100 & 100 & & & & & & & & 100 \\
\hline & & & & 0.05 & 100 & 100 & 100 & & & & & & & & 100 \\
\hline & & & & 0.1 & 100 & 100 & 100 & & & & & & & & 100 \\
\hline & & & 50 & 0.01 & 100 & 100 & 100 & 100 & 100 & 100 & & & & & 100 \\
\hline & & & & 0.05 & 100 & 100 & 100 & 100 & 100 & 100 & & & & & 100 \\
\hline & & & & 0.1 & 100 & 100 & 100 & 100 & 100 & 100 & & & & & 100 \\
\hline & & & 100 & 0.01 & 100 & 100 & 100 & 100 & 100 & 100 & 100 & 100 & 100 & 100 & 100 \\
\hline & & & & 0.05 & 100 & 100 & 100 & 100 & 100 & 100 & 100 & 100 & 100 & 100 & 100 \\
\hline & & & & 0.1 & 100 & 100 & 100 & 100 & 100 & 100 & 100 & 100 & 100 & 100 & 100 \\
\hline & & NNTS & 20 & 0.01 & 100 & 100 & 100 & & & & & & & & 100 \\
\hline & & & & 0.05 & 100 & 100 & 100 & & & & & & & & 100 \\
\hline & & & & 0.1 & 100 & 100 & 100 & & & & & & & & 100 \\
\hline & & & 50 & 0.01 & 100 & 100 & 100 & 100 & 100 & 100 & & & & & 100 \\
\hline & & & & 0.05 & 100 & 100 & 100 & 100 & 100 & 100 & & & & & 100 \\
\hline & & & & 0.1 & 100 & 100 & 100 & 100 & 100 & 100 & & & & & 100 \\
\hline & & & 100 & 0.01 & 100 & 100 & 100 & 100 & 100 & 100 & 100 & 100 & 100 & 100 & 100 \\
\hline & & & & 0.05 & 100 & 100 & 100 & 100 & 100 & 100 & 100 & 100 & 100 & 100 & 100 \\
\hline & & & & 0.1 & 100 & 100 & 100 & 100 & 100 & 100 & 100 & 100 & 100 & 100 & 100 \\
\hline von Mises & 20 & NNTS & 20 & 0.01 & 0 & 0 & 0 & & & & & & & & 2 \\
\hline & & & & 0.05 & 5 & 3 & 5 & & & & & & & & 12 \\
\hline & & & & 0.1 & 7 & 4 & 10 & & & & & & & & 23 \\
\hline & & & 50 & 0.01 & 1 & 0 & 3 & & & & & & & & 5 \\
\hline & & & & 0.05 & 3 & 1 & 10 & & & & & & & & 12 \\
\hline & & & & 0.1 & 9 & 8 & 18 & & & & & & & & 17 \\
\hline & & & 100 & 0.01 & 0 & 0 & 2 & & & & & & & & 3 \\
\hline & & & & 0.05 & 1 & 2 & 15 & & & & & & & & 15 \\
\hline & & & & 0.1 & 7 & 7 & 29 & & & & & & & & 20 \\
\hline von Mises & 50 & NNTS & 20 & 0.01 & 0 & 1 & 2 & & & & & & & & 2 \\
\hline & & & & 0.05 & 7 & 6 & 16 & & & & & & & & 15 \\
\hline & & & & 0.1 & 18 & 12 & 27 & & & & & & & & 20 \\
\hline & & & 50 & 0.01 & 3 & 2 & 11 & 18 & 14 & 12 & & & & & 5 \\
\hline & & & & 0.05 & 10 & 14 & 29 & 34 & 27 & 30 & & & & & 23 \\
\hline & & & & 0.1 & 16 & 24 & 46 & 50 & 41 & 36 & & & & & 28 \\
\hline & & & 100 & 0.01 & 3 & 6 & 24 & 32 & 38 & 17 & & & & & 12 \\
\hline & & & & 0.05 & 11 & 27 & 45 & 59 & 55 & 48 & & & & & 28 \\
\hline & & & & 0.1 & 24 & 33 & 64 & 68 & 66 & 59 & & & & & 41 \\
\hline von Mises & 100 & NNTS & 20 & 0.01 & 3 & 1 & 3 & & & & & & & & 4 \\
\hline & & & & 0.05 & 11 & 7 & 7 & & & & & & & & 13 \\
\hline & & & & 0.1 & 19 & 12 & 25 & & & & & & & & 22 \\
\hline & & & 50 & 0.01 & 4 & 8 & 17 & 19 & 17 & 19 & & & & & 7 \\
\hline & & & & 0.05 & 17 & 19 & 41 & 47 & 38 & 30 & & & & & 26 \\
\hline & & & & 0.1 & 28 & 30 & 49 & 59 & 53 & 49 & & & & & 32 \\
\hline & & & 100 & 0.01 & 9 & 11 & 32 & 50 & 42 & 40 & 30 & 27 & 24 & 19 & 11 \\
\hline & & & & 0.05 & 20 & 30 & 59 & 69 & 67 & 65 & 60 & 57 & 47 & 46 & 29 \\
\hline & & & & 0.1 & 28 & 40 & 72 & 81 & 79 & 74 & 68 & 66 & 65 & 59 & 42 \\
\hline
\end{tabular}

Table 10: NNTS homogeneity test: Statistical power. 
Citation: Fernandez-Duran JJ, Gregorio-Dominguez MM (2010) A Likelihood Ratio Test for Homogeneity in Circular Data. J Biomet Biostat 1:107. doi:10.4172/2155-6180.1000107

Page 6 of 9

\begin{tabular}{|c|c|c|c|c|c|c|c|c|c|c|c|c|c|c|c|c|}
\hline \multirow{3}{*}{$\begin{array}{c}\text { Model } \\
\text { Uniform }\end{array}$} & & & & \multicolumn{10}{|c|}{ NNTS test } & \multirow[b]{2}{*}{ Watson } & \multirow[b]{2}{*}{ Rao Mean } & \multirow[b]{2}{*}{ Rao Disp. } \\
\hline & $n_{1}$ & $n_{2}$ & $\alpha$ & $M_{0}=1$ & 2 & 3 & 4 & 5 & 6 & 7 & 8 & 9 & 10 & & & \\
\hline & 20 & 20 & 0.01 & 0 & 0 & 0 & & & & & & & & 3 & 1 & 2 \\
\hline & & & 0.05 & 0 & 0 & 0 & & & & & & & & 6 & 3 & 9 \\
\hline & & & 0.1 & 0 & 0 & 0 & & & & & & & & 10 & 8 & 13 \\
\hline & 20 & 50 & 0.01 & 0 & 0 & 0 & & & & & & & & 2 & 2 & 3 \\
\hline & & & 0.05 & 0 & 0 & 0 & & & & & & & & 4 & 5 & 9 \\
\hline & & & 0.1 & 0 & 0 & 0 & & & & & & & & 10 & 11 & 14 \\
\hline & 20 & 100 & 0.01 & 0 & 0 & 0 & & & & & & & & 2 & 0 & 5 \\
\hline & & & 0.05 & 0 & 0 & 0 & & & & & & & & 6 & 3 & 10 \\
\hline & & & 0.1 & 0 & 0 & 0 & & & & & & & & 11 & 8 & 14 \\
\hline & 50 & 50 & 0.01 & 0 & 0 & 0 & 0 & 0 & 0 & & & & & 0 & 0 & 0 \\
\hline & & & 0.05 & 0 & 0 & 0 & 0 & 0 & 0 & & & & & 4 & 7 & 3 \\
\hline & & & 0.1 & 0 & 0 & 0 & 0 & 0 & 0 & & & & & 7 & 14 & 9 \\
\hline & 50 & 100 & 0.01 & 0 & 0 & 0 & 0 & 0 & 0 & & & & & 1 & 2 & 2 \\
\hline & & & 0.05 & 0 & 0 & 0 & 0 & 0 & 0 & & & & & 2 & 5 & 4 \\
\hline & & & 0.1 & 0 & 0 & 0 & 0 & 0 & 0 & & & & & 7 & 8 & 9 \\
\hline & 100 & 100 & 0.01 & 0 & 0 & 0 & 0 & 0 & 0 & 0 & 0 & 0 & 0 & 1 & 1 & 1 \\
\hline & & & 0.05 & 0 & 0 & 0 & 0 & 0 & 0 & 0 & 0 & 0 & 0 & 4 & 8 & 4 \\
\hline & & & 0.1 & 0 & 0 & 0 & 0 & 0 & 0 & 0 & 0 & 0 & 0 & 10 & 13 & 10 \\
\hline von Mises & 20 & 20 & 0.01 & 0 & 1 & 0 & & & & & & & & 1 & 2 & 0 \\
\hline & & & 0.05 & 2 & 4 & 1 & & & & & & & & 8 & 5 & 7 \\
\hline & & & 0.1 & 2 & 6 & 5 & & & & & & & & 11 & 7 & 13 \\
\hline & 20 & 50 & 0.01 & 0 & 1 & 0 & & & & & & & & 1 & 2 & 0 \\
\hline & & & 0.05 & 4 & 2 & 2 & & & & & & & & 3 & 5 & 5 \\
\hline & & & 0.1 & 9 & 5 & 5 & & & & & & & & 9 & 6 & 11 \\
\hline & 20 & 100 & 0.01 & 0 & 0 & 0 & & & & & & & & 2 & 0 & 5 \\
\hline & & & 0.05 & 1 & 2 & 3 & & & & & & & & 7 & 2 & 8 \\
\hline & & & 0.1 & 2 & 8 & 4 & & & & & & & & 11 & 5 & 11 \\
\hline & 50 & 50 & 0.01 & 0 & 0 & 0 & 0 & 0 & 1 & & & & & 0 & 0 & 5 \\
\hline & & & 0.05 & 1 & 3 & 3 & 5 & 3 & 2 & & & & & 7 & 2 & 8 \\
\hline & & & 0.1 & 6 & 3 & 9 & 8 & 5 & 9 & & & & & 13 & 5 & 9 \\
\hline & 50 & 100 & 0.01 & 2 & 3 & 1 & 1 & 1 & 0 & & & & & 1 & 1 & 2 \\
\hline & & & 0.05 & 6 & 8 & 9 & 3 & 5 & 4 & & & & & 9 & 4 & 7 \\
\hline & & & 0.1 & 11 & 10 & 13 & 14 & 14 & 10 & & & & & 13 & 10 & 14 \\
\hline & 100 & 100 & 0.01 & 1 & 0 & 0 & 1 & 0 & 0 & 0 & 1 & 4 & 4 & 2 & 0 & 1 \\
\hline & & & 0.05 & 6 & 7 & 5 & 3 & 4 & 4 & 3 & 5 & 6 & 9 & 8 & 5 & 3 \\
\hline & & & 0.1 & 11 & 13 & 14 & 11 & 11 & 7 & 10 & 8 & 10 & 10 & 12 & 11 & 11 \\
\hline NNTS & 20 & 20 & 0.01 & 1 & 0 & 1 & & & & & & & & 1 & 0 & 2 \\
\hline & & & 0.05 & 6 & 3 & 2 & & & & & & & & 6 & 6 & 8 \\
\hline & & & 0.1 & 12 & 8 & 6 & & & & & & & & 9 & 11 & 15 \\
\hline & 20 & 50 & 0.01 & 1 & 0 & 0 & & & & & & & & 2 & 0 & 7 \\
\hline & & & 0.05 & 6 & 1 & 7 & & & & & & & & 8 & 4 & 12 \\
\hline & & & 0.1 & 7 & 5 & 11 & & & & & & & & 10 & 8 & 16 \\
\hline & 20 & 100 & 0.01 & 0 & 0 & 0 & & & & & & & & 0 & 0 & 5 \\
\hline & & & 0.05 & 3 & 1 & 1 & & & & & & & & 2 & 2 & 9 \\
\hline & & & 0.1 & 7 & 3 & 5 & & & & & & & & 8 & 12 & 17 \\
\hline & 50 & 50 & 0.01 & 4 & 0 & 0 & 1 & 1 & 1 & & & & & 3 & 0 & 3 \\
\hline & & & 0.05 & 9 & 6 & 5 & 7 & 4 & 2 & & & & & 7 & 6 & 11 \\
\hline & & & 0.1 & 17 & 14 & 14 & 12 & 10 & 5 & & & & & 14 & 14 & 15 \\
\hline & 50 & 100 & 0.01 & 3 & 0 & 0 & 0 & 0 & 1 & & & & & 0 & 0 & 1 \\
\hline & & & 0.05 & 5 & 6 & 8 & 7 & 6 & 2 & & & & & 6 & 7 & 10 \\
\hline & & & 0.1 & 10 & 16 & 11 & 12 & 8 & 9 & & & & & 11 & 13 & 13 \\
\hline & 100 & 100 & 0.01 & 1 & 0 & 1 & 0 & 2 & 1 & 3 & 3 & 1 & 3 & 0 & 0 & 0 \\
\hline & & & 0.05 & 4 & 2 & 3 & 4 & $\overline{7}$ & 5 & 5 & 8 & 4 & 5 & 8 & 2 & 8 \\
\hline & & & 0.1 & 11 & 6 & 7 & 9 & 14 & 12 & 15 & 14 & 12 & 11 & 12 & 8 & 15 \\
\hline
\end{tabular}

Table 11: NNTS homogeneity test: Type I errors

circular distribution, a von Mises distribution with $\mu=3.1962$, and $\kappa$ $=1.5510$, and NNTS density with $M=4$. Figure 1 presents the graphs for the considered models. We generated 100 simulated datasets of sample sizes equal to 20,50, and 100 for each of the three models. When comparing the simulated datasets from the different models, we studied the power of the test (the probability that the test will reject a false null hypothesis of homogeneity), and when comparing the simulated datasets from the same model, we studied the type I error of the test (the probability that the test will reject a true null hypothesis of homogeneity).

Table 10 contains the values of the power of the test that were obtained from simulating 100 different datasets for the considered models, applying the NNTS homogeneity likelihood ratio test and counting the number of times that the NNTS test correctly did not reject the null hypothesis of homogeneity for the different values of $M_{0}$ and the statistical significance of $1 \%, 5 \%$ and $10 \%$. For comparison purposes, we also applied Watson's two-sample test of homogeneity and Rao's test of homogeneity for the mean directions and dispersions using the R library circular [30]. When we tested a uniform population against a von Mises or NNTS population, the NNTS and Watson's tests gave $100 \%$ correct decisions, but Rao's test had a lower level of power specifically when testing for the equality of mean directions. The results when using Rao's test are a consequence of the fact that the mean values of the simulated models are very similar (see Figure 1); however, there are also cases with low power when testing for the equality of dispersions using Rao's test. When comparing von Mises and NNTS populations in two datasets that have a sample size equal to 20, Watson and Rao's tests have higher power than the NNTS test. By increasing the sample sizes, the power of the NNTS test increases much more quickly than the Watson's test and Rao's test. When both sample sizes are equal to 100, the NNTS has a much higher power than Watson's test and Rao's test. Generally, the maximum power the NNTS test is obtained when $M_{0}=4$, which corresponds to the true $M$ value.

Table 11 contains the type I errors that were obtained after simulating 100 different datasets of the considered models, applying the NNTS homogeneity likelihood ratio test, and counting the number of times that the NNTS test erroneously rejected the null hypothesis of homogeneity for the different values of $M_{0}$. For comparison, we 
Citation: Fernandez-Duran JJ, Gregorio-Dominguez MM (2010) A Likelihood Ratio Test for Homogeneity in Circular Data. J Biomet Biostat 1:107. doi:10.4172/2155-6180.1000107

Page 7 of 9

\begin{tabular}{|c|c|c|}
\hline & \multicolumn{2}{|c|}{ Probabilities } \\
\hline Subinterval & Model 1 & 0.12739726 \\
\hline$(0.000,0.833)$ & 0.08549016 & 0.14314715 \\
\hline$(0.833,0.167)$ & 0.08922589 & 0.16986301 \\
\hline$(0.167,0.250)$ & 0.09138272 & 0.15337195 \\
\hline$(0.250,0.333)$ & 0.09138272 & 0.12739726 \\
\hline$(0.333,0.417)$ & 0.08922589 & 0.08219178 \\
\hline$(0.417,0.500)$ & 0.08549016 & 0.04246575 \\
\hline$(0.500,0.583)$ & 0.08117651 & 0.01137866 \\
\hline$(0.583,0.667)$ & 0.07744078 & 0.00000000 \\
\hline$(0.667,0.750)$ & 0.07528395 & 0.01137866 \\
\hline$(0.750,0.833)$ & 0.07528395 & 0.04109589 \\
\hline$(0.833,0.917)$ & 0.07744078 & 0.08493151 \\
\hline
\end{tabular}

Table 12: Probabilities of the simulated models for the grouped data.

\begin{tabular}{|c|c|c|c|c|c|c|c|c|}
\hline & & & & & NNT & & & \\
\hline$n_{1}$ & $n_{2}$ & $\alpha$ & $M_{0}=1$ & 2 & 3 & 4 & 5 & 6 \\
\hline \multirow[t]{10}{*}{20} & 20 & 0.01 & 30 & 21 & 14 & 10 & 8 & 3 \\
\hline & & 0.05 & 51 & 42 & 38 & 23 & 20 & 15 \\
\hline & & 0.1 & 66 & 53 & 48 & 46 & 35 & 26 \\
\hline & 50 & 0.01 & 56 & 44 & 33 & 30 & 20 & 16 \\
\hline & & 0.05 & 76 & 66 & 57 & 52 & 46 & 40 \\
\hline & & 0.1 & 87 & 71 & 69 & 65 & 59 & 54 \\
\hline & & & & & & & & \\
\hline & 100 & 0.01 & 75 & 62 & $\frac{52}{70}$ & 50 & 37 & 30 \\
\hline & & 0.05 & 91 & 81 & 79 & 74 & 66 & 58 \\
\hline & & 0.1 & 93 & 88 & 89 & 83 & 78 & 72 \\
\hline \multirow[t]{10}{*}{50} & 20 & 0.01 & 41 & 24 & 13 & 10 & 8 & 3 \\
\hline & & 0.05 & 73 & 48 & 48 & 41 & 30 & 18 \\
\hline & & 0.1 & 86 & 68 & 58 & 52 & 47 & 36 \\
\hline & 50 & 0.01 & 86 & 71 & 58 & 51 & 50 & 43 \\
\hline & & 0.05 & 92 & 88 & 82 & 75 & 69 & 62 \\
\hline & & 0.1 & 96 & 93 & 88 & 89 & 80 & 75 \\
\hline & & & & & & & & \\
\hline & 100 & 0.01 & 98 & 93 & 85 & 84 & 81 & 76 \\
\hline & & 0.05 & 100 & 98 & 96 & 97 & 94 & 89 \\
\hline & & 0.1 & 100 & 100 & 99 & 98 & 97 & 95 \\
\hline \multirow[t]{10}{*}{100} & 20 & 0.01 & 51 & 28 & 23 & 15 & 10 & 8 \\
\hline & & 0.05 & 81 & 59 & 47 & 39 & 35 & 28 \\
\hline & & 0.1 & 93 & 77 & 66 & 58 & 47 & 39 \\
\hline & 50 & 0.01 & 97 & 87 & 81 & 72 & 66 & 56 \\
\hline & & 0.05 & 99 & 97 & 94 & 92 & 86 & 80 \\
\hline & & 0.1 & 100 & 99 & 99 & 95 & 94 & 90 \\
\hline & & & & & & & & \\
\hline & 100 & 0.01 & 100 & 100 & 99 & 97 & 96 & 92 \\
\hline & & 0.05 & 100 & 100 & 100 & 99 & 99 & 98 \\
\hline & & 0.1 & 100 & 100 & 100 & 100 & 100 & 99 \\
\hline
\end{tabular}

Table 13: NNTS homogeneity test for grouped data: Statistical power.

also applied Watson's two-sample test of homogeneity and Rao's test of homogeneity to the mean directions and dispersions. When testing a uniform population against another uniform population, the NNTS yielded probabilities of a type I errors that were equal to zero. In contrast, the Watson and Rao's tests had higher probabilities of type I errors. When applying the homogeneity tests to the datasets that were simulated from the von Mises density, the type I errors for the NNTS tests were similar or smaller than the errors from the Watson and Rao's tests. Lastly, when testing for homogeneity in the NNTS populations, the type I errors of the NNTS test and Watson and Rao's tests are similar. Note that the type I errors of the NNTS test were congruent with the significance levels used to perform the test.

For the grouped data, we simulated 100 datasets of sizes 20, 50, and 100 for two circular populations in the interval $(0,1)$ that were partitioned on subintervals of equal length $\frac{1}{12}$. Table 12 presents the probabilities for each model. For Model 1, the probabilities were obtained using the formula for a simple sinusoidal curve [31] $P_{k}=\frac{1+0.1 \sin \left(\frac{(2 k-1) \pi}{12}\right)}{12}$, and for Model 2, $P_{k}=\frac{1+0.25 \sin \left(\frac{k \pi}{6}\right)}{12}$ [32] for $k=1, \ldots, 12$ were used. Based on the results in Tables 13 and 14 , where $n_{1}$ and $n$ are the number of observations that were simulated from models 1 and 2 , respectively, the NNTS test for homogeneity presented good power and type I errors that were congruent with the considered significance levels. As expected, the NNTS model with $M_{0}=1$ presented the best power because we were simulating from simple sinusoidal models.

\section{Conclusions}

The family of circular distributions that is based on nonnegative trigonometric sums (NNTS) is able to model circular populations that present multimodality and/or skewness. This flexibility of the NNTS models makes them suitable candidates for the construction of homogeneity tests for circular data when samples from different circular populations are used to test the equality of the populations 
Citation: Fernandez-Duran JJ, Gregorio-Dominguez MM (2010) A Likelihood Ratio Test for Homogeneity in Circular Data. J Biomet Biostat 1:107. doi:10.4172/2155-6180.1000107

Page 8 of 9

\begin{tabular}{|c|c|c|c|c|c|c|c|c|}
\hline & & & & & $\mathrm{NN}$ & & & \\
\hline$n_{1}$ & $n_{2}$ & $\alpha$ & $M_{0}=1$ & 2 & 3 & 4 & 5 & 6 \\
\hline \multirow[t]{3}{*}{20} & 20 & 0.01 & 1 & 2 & 1 & 0 & 0 & 0 \\
\hline & & 0.05 & 6 & 7 & 5 & 8 & 2 & 1 \\
\hline & & 0.1 & 9 & 10 & 12 & 12 & 9 & 4 \\
\hline \multirow[t]{3}{*}{20} & 50 & 0.01 & 1 & 0 & 0 & 0 & 0 & 0 \\
\hline & & 0.05 & 4 & 4 & 3 & 3 & 4 & 0 \\
\hline & & 0.1 & 6 & 11 & 11 & 8 & 6 & 5 \\
\hline & & & & & & & & \\
\hline \multirow[t]{3}{*}{20} & 100 & 0.01 & 0 & 0 & 0 & 0 & 0 & 0 \\
\hline & & 0.05 & 1 & 2 & 1 & 0 & 0 & 0 \\
\hline & & 0.1 & 3 & 5 & 3 & 3 & 3 & 0 \\
\hline \multirow[t]{3}{*}{50} & 50 & 0.01 & 1 & 3 & 2 & 2 & 3 & 1 \\
\hline & & 0.05 & 4 & 6 & 12 & 12 & 10 & 7 \\
\hline & & 0.1 & 14 & 9 & 16 & 19 & 18 & 13 \\
\hline & & & & & & & & \\
\hline \multirow[t]{3}{*}{50} & 100 & 0.01 & 1 & 0 & 0 & 0 & 0 & 0 \\
\hline & & 0.05 & 4 & 3 & 1 & 2 & 2 & 1 \\
\hline & & 0.1 & 10 & 10 & 8 & 7 & 6 & 3 \\
\hline \multirow{4}{*}{100} & & & & & & & & \\
\hline & 100 & 0.01 & 3 & 4 & 5 & 6 & 5 & 4 \\
\hline & & 0.05 & 7 & 7 & 8 & 9 & 8 & 7 \\
\hline & & 0.1 & 11 & 10 & 11 & 11 & 13 & 10 \\
\hline & & & & & & & & \\
\hline \multirow[t]{3}{*}{20} & 20 & 0.01 & 0 & 1 & 0 & 0 & 0 & 0 \\
\hline & & 0.05 & 1 & 4 & 2 & 2 & 1 & 1 \\
\hline & & 0.1 & 3 & 5 & 3 & 2 & 2 & 1 \\
\hline & & & & & & & & \\
\hline \multirow[t]{3}{*}{20} & 50 & 0.01 & 0 & 0 & 0 & 0 & 0 & 0 \\
\hline & & 0.05 & 3 & 1 & 1 & 1 & 0 & 0 \\
\hline & & 0.1 & 5 & 5 & 4 & 3 & 2 & 0 \\
\hline \multirow[t]{3}{*}{20} & 100 & 0.01 & 0 & 0 & 0 & 0 & 0 & 0 \\
\hline & & 0.05 & 1 & 2 & 1 & 0 & 0 & 0 \\
\hline & & 0.1 & 3 & 5 & 3 & 3 & 3 & 0 \\
\hline & & & & & & & & \\
\hline \multirow[t]{3}{*}{50} & 50 & 0.01 & 0 & 0 & 0 & 0 & 0 & 0 \\
\hline & & 0.05 & 0 & 0 & 1 & 1 & 2 & 1 \\
\hline & & 0.1 & 2 & 2 & 3 & 3 & 3 & 3 \\
\hline \multirow[t]{3}{*}{50} & 100 & 0.01 & 0 & 1 & 0 & 1 & 0 & 0 \\
\hline & & 0.05 & 2 & 1 & 2 & 4 & 3 & 1 \\
\hline & & 0.1 & 5 & 3 & 2 & 4 & 4 & 4 \\
\hline & & & & & & & & \\
\hline \multirow[t]{3}{*}{100} & 100 & 0.01 & 1 & 0 & 1 & 0 & 0 & 0 \\
\hline & & 0.05 & 4 & 3 & 4 & 1 & 1 & 0 \\
\hline & & 0.1 & 4 & 5 & 6 & 7 & 2 & 2 \\
\hline
\end{tabular}

Table 14: NNTS homogeneity test for grouped data: Type I Errors.

circular distributions. In this paper, a likelihood ratio homogeneity test for circular data was constructed by considering that the distributions of the different populations are or can be approximated by members of the NNTS family. Importantly, this test can be applied to ungrouped or grouped observations from these populations. The test was applied to simulated data, confirming the suitability of the test. Also, the test was applied to interesting real datasets to obtain conclusions about the homogeneity of the circular populations. A simulation study showed that the type I error and the power of the proposed test are similar or better than those of the Watson's test and Rao's test for the tested populations (uniform, von Mises and NNTS).

\section{Acknowledgements}

The authors thank the referees for their useful comments and are grateful to the Asociación Mexicana de Cultura A.C. for its support.

\section{References}

1. Fernandez-Duran JJ (2004) Circular Distributions Based on Nonnegative Trigonometric Sums. Biometrics 60: 499-503.

2. Fejer $L$ (1916) Uber Trigonometrische Polynome. Journal fur die Reine und Angewandte Mathematik. 146: 53-82.

3. Fernandez-Duran JJ (2007) Models for Circular-Linear and CircularCircular Data Constructed from Circular Distributions Based on Nonnegative Trigonometric Sums. Biometrics 63: 579-585.

4. Fernandez-Duran JJ, Gregorio-Dominguez MM (2010) Maximum Likelihood
Estimation of Nonnegative Trigonometric Sum Models Using a Newton-like Algorithm on Manifolds. arXiV:1010.6098v1 [stat.CO].

5. R Development Core Team (2010) R: A language and environment fo statistical computing, ISBN 3-900051-07-0, R Foundation for Statistical Computing,Vienna, Austria.

6. Fernandez-Duran JJ, Gregorio-Dominguez MM (2009) CircNNTSR: An R Package for the Statistical Analysis of Circular Data Using Nonnegative Trigonometric Sums (NNTS) Models v0.1. Working Paper, DE-C09-13, Department of Statistics, ITAM, Mexico.

7. Fernandez-Duran JJ Gregorio-Dominguez MM (2010) CircNNTSR: An $\mathrm{R}$ package for the statistical analysis of circular data using nonnegative trigonometric sums (NNTS) models. R package version 1.0-1.

8. Mardia KV, Spurr BD (1973) Multisample Tests for Multimodal and Axial Circular Populations. J R Stat Soc Series B Stat Methodol 35: 422-436.

9. Mardia KV (1972) Statistics of Directional Data. Academic Press, London

10. Wheeler S, Watson GS (1964) A Distribution-Free Two-Sample Test on the Circle. Biometrika 51: 256-257.

11. Rao JS (1967) Large Sample Tests for the Homogeneity of Angular Data. Sankhya 28: 172-174.

12. Rao JS (1984) Nonparametric Methods in Directional Data. Chapter 31 in Handbook of Statistics, Volume 4. Nonparametric Statistics, P.R. Krishnaiah and P.K. Sen (eds.), pp. 755-770. Amsterdam: Elsevier Science Publishers.

13. Jammalamadaka SR, SenGupta A (2001) Topics in Circular Statistics.: World Scientific Publishing, Co., Singapore. 
Citation: Fernandez-Duran JJ, Gregorio-Dominguez MM (2010) A Likelihood Ratio Test for Homogeneity in Circular Data. J Biomet Biostat 1:107. doi:10.4172/2155-6180.1000107

Page 9 of 9

14. Watson GS (1962) Goodness-of-fit Tests on a Circle, II. Biometrika 49: 57-63.

15. Watson GS, Williams EJ (1956) On the Construction of Significance Tests on the Circle and the Sphere. Biometrika 43: 344-352.

16. Stephens MA (1969) Tests for the von Mises Distribution. Biometrika 56: 149160.

17. Watson GS (1983) Statistics on Spheres. Wiley, New York.

18. Fisher NI, Hall P (1990) New Statistical Methods for Directional Data - I. Bootstrap Comparison of Mean Directions and the Fold Test in Palaeomagnetism. Geophysical Journal International 101: 305-313.

19. Harrison D, Kanji GK, Gadsden RJ (1986) Analysis of Variance for Circular Data. J Appl Stat 13: 123-138.

20. Harrison D, Kanji GK (1988) The Development of Analysis of Variance for Circular Data. J Appl Stat 15: 197-224.

21. Stephens MA (1992) On Watson's ANOVA for Directions. In K.V. Mardia (ed.) The Art of Statistical Science: A Tribute to G.S. Watson, pp. 75-85, Chichester: Wiley.

22. Anderson CM, Wu CFJ (1995) Measuring Location Effects from Factorial Experiments with a Directional Response. Int Stat Rev 63: 345-363.

23. Grimshaw SD, Whiting DG, Morris TH (2001) Likelihood Ratio Tests for a Mixture of Two von Mises Distributions. Biometrics 57: 260-265.
24. Chen J, Li P, Fu Y (2008) Testing Homogeneity in a Mixture of von Mises Distributions with a Structural Parameter. Can J Stat 36: 129-142.

25. Fu Y, Chen J, Li P (2008) Modified Likelihood Ratio Test for Homogeneity in a Mixture of von Mises Distributions. J Stat Plan Inference 138: 667-681.

26. Mardia KV, Jupp PE (2000) Directional Statistics. (2Edition), John Wiley and Sons, Great Britain.

27. Mardia KV (1972) A Multi-Sample Uniform Scores Test on a Circle and its Parametric Competitor. J R Stat Soc Series B Stat Methodol 34: 102-113.

28. Fisher NI (1993) Statistical Analysis of Circular Data. Cambridge: Cambridge University Press

29. Wells NA (1990) Comparing Sets of Circular Orientations by Modified Chisquared Testing. Comput Geosci 16: 1155-1170.

30. Lund U, Agostinelli C (2010) circular: Circular Statistics. R package version 0.4

31. Marrero O (1983) The Performance of Several Statistical Tests for Seasonality in Monthly Data. Journal of Statistical Computation and Simulation 17: 275-296.

32. Freedman LS (1979) The Use of a Kolmogorow-Smirnov Type Statistic in Testing Hypotheses about Seasonal Variation. J Epidemiol Community Health 33: $223-228$ 